\title{
23andMe wades further into drug discovery
}

Direct-to-consumer genetics testing company 23 andMe is advancing its drug discovery efforts with a $\$ 250$ million financing round announced in September. The Mountain View, California-based firm plans to use the funds for its own therapeutics division aimed at mining the company's database for novel drug targets, in addition to its existing consumer genomics business and genetic research platform. At the same time, the company has strengthened ongoing partnerships with Pfizer and Roche, and inked a new collaboration with Lundbeckall are keen to incorporate 23andMe's human genetics data cache into their discovery and clinical programs.

It was over a decade ago that Icelandic company deCODE Genetics pioneered genetics-driven drug discovery. The Reykjavik-based biotech's DNA database of 140,000 Icelanders, which Amgen bought in 2012 (Nat. Biotechnol. 31, 87-88, 2013), was set up to identify genes associated with disease. But whereas the bedrock of deCODE's platform was the health records stretching back over a century, the value in 23 andMe's platform lies instead in its database of more

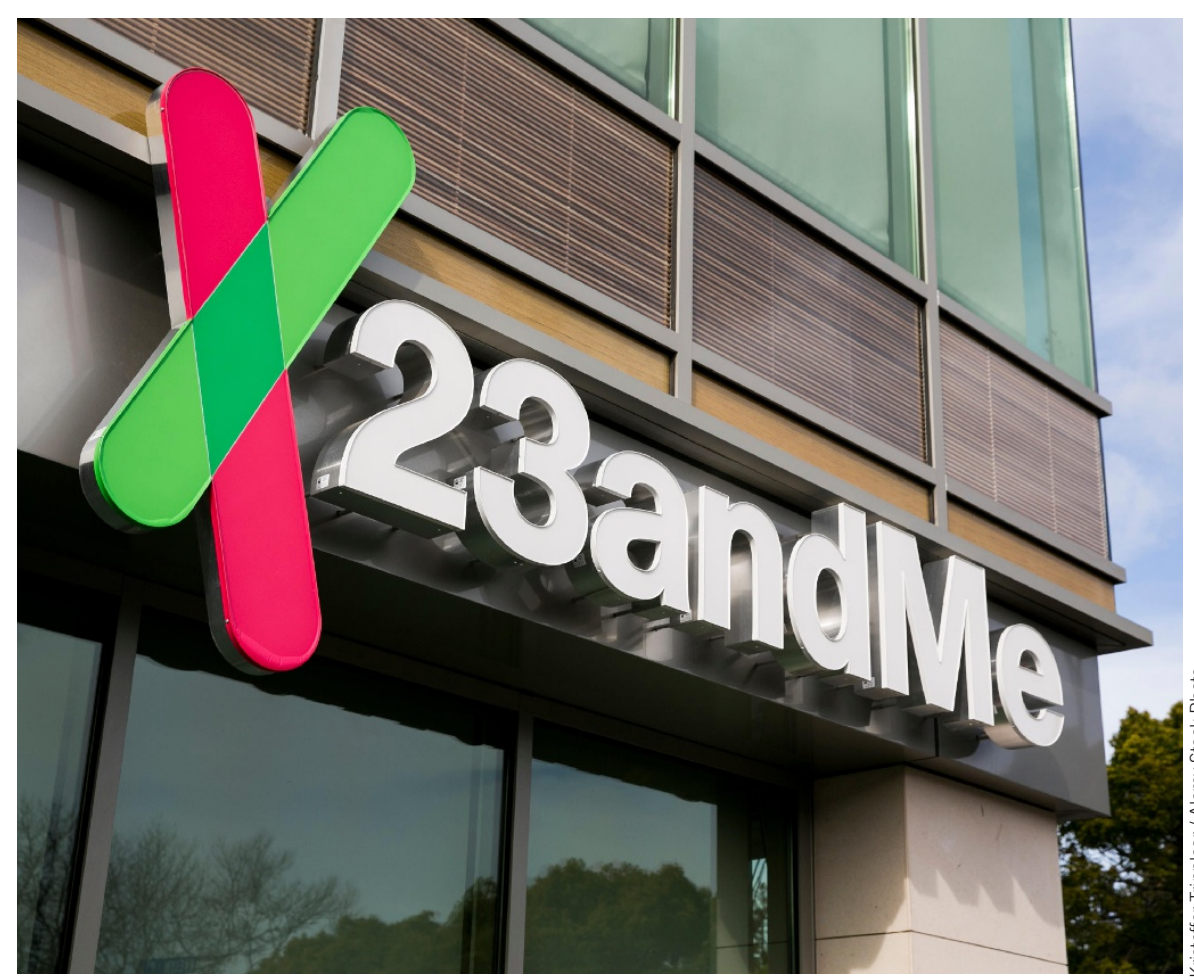

Beyond consumer genetics: 23andMe sells access to its database to drug companies.

than 2 million genotyped customers, and the reams of phenotypic information participants collect at home by online surveys of mood, cognition and even food intake.

For Danish pharma Lundbeck, a partnership signed in August with 23andMe and think-tank Milken Institute will provide a fresh look at major depressive disorder and bipolar depression. The collaboration studying 25,000 participants will link genomics with complete cognitive tests and surveys taken over nine months, providing an almost continuous monitoring of participants' symptoms. "Cognition is a key symptom in depression," says Niels Plath, vice president for synaptic transmission at Copenhagenbased Lundbeck. But the biological processes leading to depression are poorly understood, and the condition is difficult to classify as it includes a broad population of patients. "If we could use genetic profiling to sort people into groups and link to biology, we could identify new drug targets, novel pathways and protein networks. With 23 andMe, we can combine the genetic profiling with symptomatic presentation," says Plath. An approach like this leapfrogs the traditional paradigm of mouse models and cell-based assays for drug discovery. "Our scientific hypotheses must come from patient-derived information," says Plath. "It could be phenotype, it could be genetic."

Drug maker Roche has been taking advantage of 23andMe's data cache for several years, and its collaborations are yielding results. In September, researchers from the Basel-based pharma's wholly owned Genentech subsidiary, in partnership with 23 andMe and others, published a paper showcasing 17 new Parkinson's disease risk loci that could be potential targets for therapeutics (Nat. Genet. http://dx.doi.org/10.1038/ng.3955, 2017).

A year earlier, in August 2016, scientists at New York-based Pfizer, 23andMe and Massachusetts General Hospital announced that they had identified 15 genetic regions linked to depression (Nat. Genet. 48, 10311036, 2016). A 23andMe spokesperson this week called that paper a "landmark," because it was the first study to uncover 17 variants associated with major depressive disorder.

Ashley Winslow, who was corresponding author on the 2016 Nature Genetics paper, and who used to work at Pfizer, says, "Initially, the focus was on using the database to either confirm [or refute] the findings established by traditional, clinical methods of ascertainment." It soon occurred to the investigators that they could move beyond traditional association studies and do discovery work in indications that to date had "not been well powered," such as major depression, especially since some of 23 andMe's questionnaires specifically asked if subjects had once been clinically diagnosed.

"I think [the database is] of particular interest for psychiatric disorders because the medications just have such a poor track record of not working," says Winslow, now senior director of translational research and portfolio development at the University of Pennsylvania's Orphan Disease Center in Philadelphia. "23andMe offered us a fresh new look."

Winslow thinks there is a "powerful shift" under way in pharma as it recognizes the benefits of rooting target discovery in human-derived data. "You still have to do the work-up through cell-line screening or animals at some point, but the starting point being human-derived data is hugely important."

Justin Petrone Tartu, Estonia 